\title{
Fertilización nitrogenada en girasol en el suroeste de Chaco
}

\author{
Cáceres Díaz, Raúl O. ; Ledda, Rosa A.²; Aranda, Juan. ${ }^{3}$ \\ 1EEA INTA Salta (caceresdiaz.raul@inta.gob.ar); ${ }^{2}$ EEA INTA Las Breñas (ledda.alejandra@inta.gob.ar); \\ ${ }^{3}$ EEA INTA Las Breñas (aranda.juan@inta.gob.ar).
}

\begin{abstract}
Resumen
La producción de girasol en la provincia de Chaco se ubica en el tercer lugar a nivel nacional, con rindes para la campaña 2010/2011 de $1860 \mathrm{~kg} \mathrm{ha}^{-1}$. En este trabajo se plantea evaluar la respuesta de la fertilización nitrogenada con urea en prefloración incorporada a dosis de 100 y $150 \mathrm{~kg} \mathrm{ha}^{-1}$ en Labranza Cero, utilizando un material considerado de alto potencial DKSOL 4045, sembrado a $0.52 \mathrm{~m}$ entre líneas y testigo sin fertilizar con tres densidades de siembra $\left(2.17 \mathrm{pl} \mathrm{m}^{-1} ; 3 \mathrm{pl} \mathrm{m}^{-1}\right.$ y $3.83 \mathrm{pl} \mathrm{x} \mathrm{m}^{-1}$ plantas logradas). Los resultados se analizaron con el software INFOGEN Ver. 2011/P. Se logró un stand de plantas promedio de 2.86 en un metro, $16.57 \mathrm{~cm}$ de diámetro de capítulo, $1.66 \mathrm{~m}$ de alto, $124.78 \mathrm{~g}$ el peso de mil semillas y un rendimiento promedio de $2348.2 \mathrm{~kg} \mathrm{ha}^{-1}$. Existe una correlación positiva entre el peso de mil semillas (0.59) diámetro de capítulo (0.55), altura de planta (0.52) y dosis de urea (0.49) con el rendimiento. Existieron diferencias estadísticas en el rendimiento a dosis creciente de urea desde testigo $1737.54 \mathrm{~kg} \mathrm{ha}^{-1}, 150 \mathrm{~kg}$ de urea ha ${ }^{-1} 2531.14 \mathrm{~kg} \mathrm{ha}^{-1}$ de rendimiento y $100 \mathrm{~kg}$ de urea $\mathrm{ha}^{-1}$ con un rendimiento de $2775.92 \mathrm{~kg} \mathrm{ha}^{-1}$. La fertilización respondió a una ecuación polinómica con $\mathrm{Y}=-0.0969 \mathrm{x}^{2}+1811.7$ con un $\mathrm{R}^{2}=0.3114$. La eficiencia de uso de urea fue de $27.75 \mathrm{~kg}$ de grano producido por $\mathrm{kg}$ de urea aplicado para $100 \mathrm{~kg}$ de urea y $16.87 \mathrm{~kg}$ de grano logrado por kg de urea aplicada en $150 \mathrm{~kg}$ de urea aplicada. El agregado de $100 \mathrm{~kg}$ de urea al inicio de floración incorporada al surco permitió incrementar los rendimientos de girasol en $59.7 \%$.
\end{abstract}

Palabras clave: labranza cero, prefloración, eficiencia uso agua, eficiencia uso urea.

\begin{abstract}
Summary
Sunflower production in the Chaco province represents the third yield al national level, with yields of fo1860 $\mathrm{kg} \mathrm{x} \mathrm{ha}^{-1}$ per 2010/2011 season. The aim of this work was to evaluate the response to nitrogen fertilization with urea in pre built at doses of 100 and $150 \mathrm{~kg} \mathrm{x} \mathrm{ha}^{-1}$ in zero tillage, using a high potential material, DKSOL 4045, at $0.52 \mathrm{~m}$ between lines and control without fertilize with three densities $\left(2.17 \mathrm{pl} \mathrm{x} \mathrm{m}^{-1} ; 3 \mathrm{pl} \mathrm{x} \mathrm{m}^{-1}\right.$ y $3.83 \mathrm{pl} \mathrm{x} \mathrm{m}^{-1}$ plants achieved). The results were analyzed with the software INFOGEN V. 2011 / P. Average plant stand was 2.86 in one meter, diameter section was $16.57 \mathrm{~cm}, 1.66$ $\mathrm{m}$ high, $124.78 \mathrm{~g}$ thousand grain weight and yield of $2348.2 \mathrm{~kg} \mathrm{x} \mathrm{ha}^{-1}$. There is a positive correlation between thousand grain weight (0.59) diameter section (0.55), plant height (0.52) and dose of urea (0.49) with yield. Statistical differences were detected related increased dose of urea, being control 1737.54 $\mathrm{kg} \mathrm{x} \mathrm{ha-1,} 150 \mathrm{~kg} \mathrm{ha}^{-1}$ urea produced $2531.14 \mathrm{~kg} \mathrm{x} \mathrm{ha}^{-1}$ and $100 \mathrm{~kg} \mathrm{x} \mathrm{ha}^{-1}$ urea with a yield of $2775.92 \mathrm{~kg}$ $\mathrm{x} \mathrm{ha} \mathrm{h}^{-1}$. Fertilization responded to a polynomial equation $\mathrm{Y}=-0.0969 \times 2+1811.7$ with $\mathrm{R}^{2}=0.3114$. The efficiency of use of urea was $27.75 \mathrm{~kg}$ of grain produced per $\mathrm{kg}$ of urea applied at a dose of $100 \mathrm{~kg}$ urea and $16.87 \mathrm{~kg}$ per $\mathrm{kg}$ grain of urea applied at $150 \mathrm{~kg}$ of urea applied.The addition of $100 \mathrm{~kg}$ of urea at the beginning of flowering incorporated to soil, at the groove, allowed increased sunflower yields $59.7 \%$. Key words: zero tillage, pre-flowering, water use efficiency, urea use efficiency.
\end{abstract}

\section{INTRODUCCIÓN}

La producción de girasol en la provincia de Chaco se ubica en el tercer lugar a nivel nacional. En la campaña 2011/12 se sembraron 215000 ha con una producción estimada de 400000 t. En la Figura $\mathbf{N}^{\mathbf{0}} \mathbf{1}$, se observan los rendimientos provinciales que se encuentran para la campaña 2011/12 en los $1860 \mathrm{~kg} \mathrm{ha}^{-1}$. Desde el año 2003 al 2011 hubo una caída en la superficie sembrada, pasando de 300000 ha a 215000 ha en toda la provincia.

En la región Suroeste de Chaco se produce el 60\% del girasol de la provincia. Experiencias realizadas en establecimientos de productores de girasol del área de la Estación Experimental Agropecuaria Las Breñas del INTA señalan que con aplicaciones de 90 a $100 \mathrm{~kg} \mathrm{ha}^{-1}$ de urea y buena disponibilidad hídrica, es posible incrementar en $300 \mathrm{~kg} \mathrm{ha}^{-1}$ los rendimientos; pero los estudios revelaron un descenso en el porcentaje de materia grasa, que oscila entre 1 y 2 puntos (Parra y Sosa, 2001).

Las condiciones ambientales sitúan al Suroeste de Chaco como una región adecuada para el cultivo, con rendimientos óptimos, considerando las escasas precipitaciones invernales promedio normales que están 


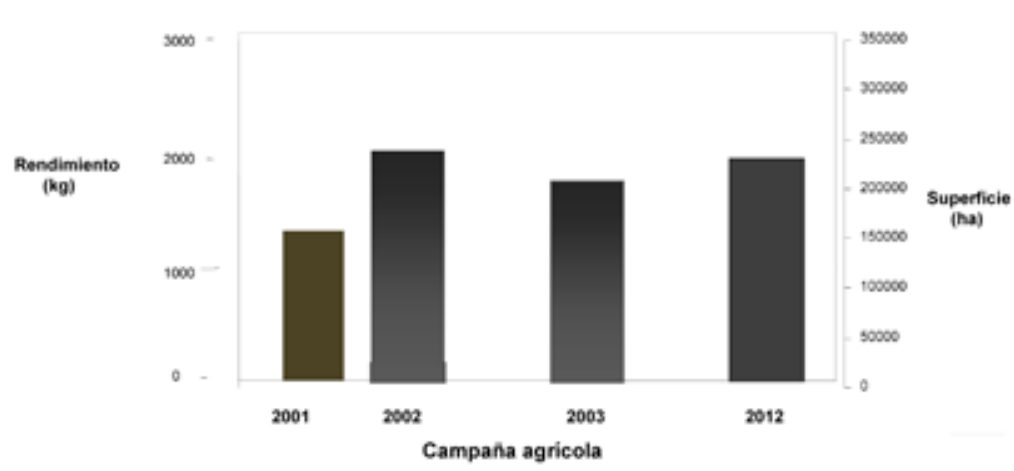

Figura $\mathbf{N}^{\mathbf{0}}$ 1. Rendimiento de girasol (kg) y superficie sembrada (ha) por campaña agrícola en Chaco.

en el orden de los $383 \mathrm{~mm}$ en el ciclo del cultivo de julio a diciembre. Halvorson et al. (1999) mencionan que una cantidad total de agua disponible menor a $300 \mathrm{~mm}$ afecta el rendimiento potencial del girasol entre 300 y $500 \mathrm{~mm}$ definen los rendimientos potenciales y superiores a $500 \mathrm{~mm}$, no afectan los rendimientos potenciales del girasol como respuesta a la labranza y la fertilización nitrogenada.

\section{MATERIALES Y MÉTODOS}

El ensayo se realizó en la Estación Experimental Agropecuaria INTA Las Breñas, Chaco con su cabecera de cuadro ubicada en $\operatorname{los} 27^{\circ} 04^{\prime} 32^{\prime \prime}$ Sur y $61^{\circ}$ $02^{\prime} 42^{\prime \prime}$ Oeste.

El suelo responde a una asociación compuesta por las series Tizón y Tanigó. El suelo de la serie Tizón está clasificada taxonómicamente como Haplustol óxico. El material originario es Loess, con relieve normal; suelos con débil desarrollo de horizontes genéticos, caracterizados por un incipiente $\mathrm{B}$, formado por meteorización in situ (grado de desarrollo 2). La serie Tanigó corresponde a un suelo Argiacuol vértico; cuyo material originario son arcillas lacustres, con relieve cóncavo. Son suelos con perfiles fuertemente desarrollados de tipo A-B-C, con cierta evidencia de formación de un A2 por encima del B textural (grado de desarrollo 5).

Se midieron $44 \mathrm{~mm}$ de lámina de agua totales a la siembra hasta $1 \mathrm{~m}$ de profundidad. El cultivo antecesor fue soja y el lote se hallaba en barbecho de 12 meses.

Se utilizó un material considerado por el semillero de alto potencial de rendimiento, el híbrido DKSOL 4045 de DEKALB Grado 2, de ciclo largo excelente comportamiento ante roya negra, verticilium y tolerante a las nuevas razas de mildiú. Se caracteriza por: 72 a 75 días a floración, color del aquenio estriado, materia grasa media a baja, tallo fuerte, estabilidad de rendimiento, muy destacado comportamiento a estrés hídrico y tolerancia al ataque de pájaros.

Se sembró en Labranza Cero a $0.52 \mathrm{~m}$ entre surcos con sembradora PLA de 8 surcos con tres densidades de siembra promedio de $2.17 \mathrm{pl} \mathrm{m}^{-1} ; 3 \mathrm{pl} \mathrm{m}{ }^{-1}$ y $3.83 \mathrm{pl}$ $\mathrm{m}^{-1}$ logradas en el surco a cosecha y dosis creciente de urea de $0 ; 100$ y $150 \mathrm{~kg} \mathrm{ha}^{-1}$, incorporada en el surco en inicio de floración, en total 9 Tratamientos distribuidos en

Bloques Completos al Azar con cuatro repeticiones. Las parcelas consistieron en 8 surcos de $4 \mathrm{~m}$ de largo, fertilizando todo el largo de la parcela y los 6 surcos centrales, incorporando en forma manual en el surco la dosis de urea evaluada.

El control de malezas se realizó mediante herbicidas preemergentes, Acetoclor (90\%) 1,5 $1 \mathrm{ha}^{-1}$, Prometrina (50\%) 1,5 $1 \mathrm{ha}^{-1}$ y Aclonifen ( 60\%) $1 \mathrm{l} \mathrm{ha}^{-1}$ postemergente.

Se cosecharon muestras centrales de cada parcela de $2 \mathrm{~m}$ de largo y 2 surcos contiguos en el centro de cada parcela con $24 \%$ de humedad. La cosecha se realizó en forma anticipada para disminuir el daño por aves a las muestras. Las muestras se llevaron, previa toma de peso a estufa a $70^{\circ} \mathrm{C}$ durante 5 días con posterior análisis de rendimiento y componentes.

Se utilizó el software INFOGEN Ver. 2011/P para el análisis estadístico de Varianza, Correlación y Análisis de Regresión.

\section{RESULTADOS Y DISCUSIÓN}

Como lo muestra la Figura $\mathbf{N}^{\mathbf{0}} \mathbf{2}$, en la campaña del ensayo se registraron $320.2 \mathrm{~mm}$ desde el $1^{\circ}$ de julio al 30 de diciembre, un $16.4 \%$ menos que lo normal. Se destaca que en el mes de agosto no ocurrieron precipitaciones, retrasando la fecha de siembra óptima a la primera semana de setiembre.

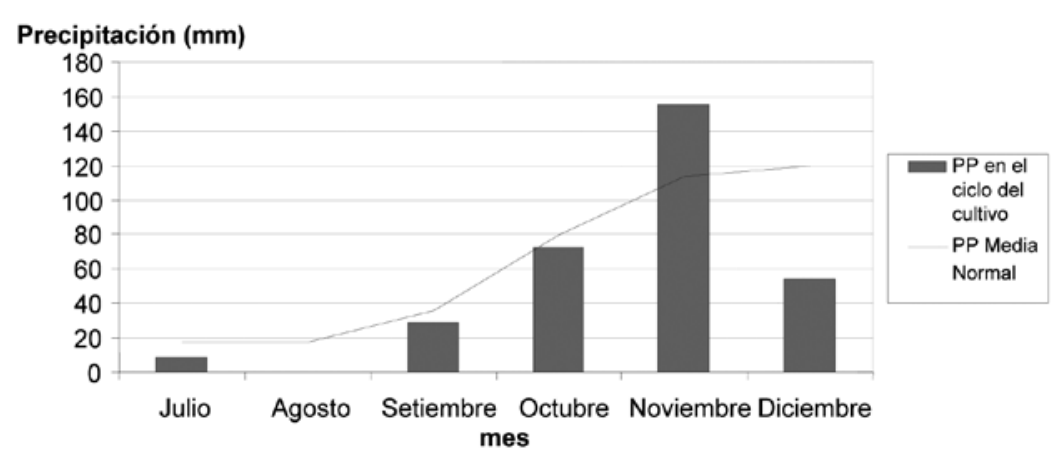

Figura $\mathbf{N}^{\circ}$ 2. Precipitaciones media normal y las ocurridas en el ciclo del cultivo de girasol, campaña 2011/2012. Fuente: Estación Agrometeorológica EEA INTA Las Breñas, 2011. 
Tabla $\mathbf{N}^{0}$ 1. Estadística descriptiva de las variables en estudio. Media, Desvío Estandar (DE), Coeficiente de Variación (CV), Mínimo (Mín) y Máximo (Máx).

\begin{tabular}{lcccccc}
\hline Variable & $\mathbf{n}$ & Media & DE & CV & Mín. & Máx. \\
\hline Plantas por metro & 36 & 2.86 & 0.63 & 21.85 & 1.75 & 4.25 \\
Diámetro de capítulo & 36 & 16.57 & 2.02 & 12.21 & 11.83 & 21.00 \\
Altura de planta (m) & 36 & 1.66 & 0.15 & 8.77 & 1.36 & 1.93 \\
Peso de mil semillas (g) & 36 & 124.78 & 22.63 & 18.14 & 87.74 & 174.24 \\
Rendimiento ajustado 11\% de humedad $\left(\mathrm{kg} \mathrm{ha}^{-1}\right)$ & 36 & 2348.2 & 769.43 & 32.77 & 975.21 & 4062.05 \\
\hline
\end{tabular}

Tabla No 2: Coeficientes de correlación Pearson entre parámetros evaluados.

\begin{tabular}{|c|c|c|c|c|c|c|}
\hline & $\begin{array}{l}\text { Rendimiento } \\
\text { a } 11 \%\end{array}$ & Plantas $\mathrm{m}^{-1}$ & $\begin{array}{l}\text { Dosis } \\
\text { urea }\end{array}$ & $\begin{array}{l}\text { Peso } 1000 \text { semillas } \\
(\mathrm{g})\end{array}$ & $\begin{array}{l}\text { Altura de } \\
\text { planta }\end{array}$ & $\begin{array}{l}\text { Diámetro } \\
\text { capítulo }\end{array}$ \\
\hline Rendimiento a $11 \%$ & 1,00 & 0,29 & $2,2 \mathrm{E}-03$ & $1,4 \mathrm{E}-04$ & $1,0 \mathrm{E}-03$ & $4,8 \mathrm{E}-04$ \\
\hline Plantas $\mathrm{m}^{-1}$ & $-0,18$ & 1,00 & 1,00 & 0,89 & 0,55 & 0,50 \\
\hline Dosis urea & 0,49 & 0,00 & 1,00 & 0,45 & 0,08 & 0,28 \\
\hline Peso 1000 semillas (g) & 0,59 & $-0,02$ & 0,13 & 1,00 & 0,54 & 5,0E-03 \\
\hline Altura de planta & 0,52 & $-0,10$ & 0,30 & 0,11 & 1,00 & 0,08 \\
\hline Diametro capítulo & 0,55 & $-0,12$ & 0,19 & 0,46 & 0,30 & 1,00 \\
\hline
\end{tabular}

En la Tabla $\mathbf{N}^{\mathbf{0}} \mathbf{1}$ se observa que existen Tratamientos con valores extremos de rendimientos de 4062.05 $\mathrm{kg} \mathrm{ha}^{-1}$, valores extremos promedios de diámetro de capítulo de $21 \mathrm{~cm}$ y peso extremo de mil semillas de $174.24 \mathrm{~g}$. Estos valores nos podrían indicar que el cultivo presenta aun, un margen para mejorar su desempeño cuando el ambiente y el manejo es apropiado (fecha de siembra, densidad, disponibilidad hídrica, nutrientes).

Los valores de altura promedio resultaron superiores en $5 \mathrm{~cm}$ a los obtenidos en los ensayos comparativos de rendimiento llevados a cabo en INTA Reconquista para el mismo cultivar, en tanto el rendimiento promedio resultó inferior al obtenido en el mismo ensayo en $385 \mathrm{~kg} \mathrm{ha}^{-1}$ (Parra y Longhi, 2010).

En la Tabla $\mathbf{N}^{\mathbf{0}} \mathbf{2}$, se observa que existe una correlación positiva entre el rendimiento y dosis de urea $(0.49)$ peso de mil semillas (0.59) altura de planta $(0.52)$ y diámetro de capítulo (0.55). Esto mismo fue reportado por Ghanavati et al. (1981) para ensayos de cruza de materiales en Irán. Existiendo una correlación negativa entre el rendimiento y la cantidad de plantas por metro (-0.18) y negativa entre pantas por metro y altura de planta $(-0.10)$ y diámetro de capítulo $(-0.12)$.

No existen diferencias significativas entre los rendimientos obtenidos para $2.17 \mathrm{pl} \mathrm{m}^{-1} ; 3 \mathrm{pl} \mathrm{m}^{-1}$ y 3.83

$\mathrm{pl} \mathrm{m}{ }^{-1}$ como lo muestra la Tabla $\mathbf{N}^{\circ} \mathbf{3}$, aunque la menor cantidad de plantas por metro $\left(2.17 \mathrm{pl} \mathrm{m}^{-1}\right)$ logró los más altos rendimientos (2621.69 $\left.\mathrm{kg} \mathrm{ha}^{-1}\right)$.

$\mathrm{Al}$ analizar los rendimientos en función de la dosis de urea aplicada se observa que existen diferencias significativas entre el testigo y las dosis de urea aplicada, no así entre las dos dosis de urea $100 \mathrm{~kg} \mathrm{ha}^{-1} \mathrm{y}$
$150 \mathrm{~kg} \mathrm{ha}^{-1}$ Tabla $\mathbf{N}^{\circ} 4$. Halvorson et al. (1999) mencionan rendimientos incrementales con fertilización nitrogenada de más de $34 \mathrm{~kg}$ de $\mathrm{N}$ para las Grandes Planicies de EUA.

Al analizar los rendimientos para el testigo, sin fertilización $\left(0 \mathrm{~kg}\right.$ de urea ha $\left.{ }^{-1}\right)$ en función de la cantidad de plantas por metro, se observa que los rendimientos son mayores 2282,98 $\mathrm{kg} \mathrm{ha}^{-1}$ cuando menor es la cantidad de plantas por metro $2.17 \mathrm{pl} \mathrm{m}^{-1}$, existiendo diferencias significativas entre los rendimientos (Tabla $\left.\mathbf{N}^{\circ} \mathbf{5}\right)$.

Tabla $N^{\circ}$ 3: Rendimientos medios $\left(\mathrm{kg} \mathrm{ha}^{-1}\right)$ obtenidos de acuerdo al número de plantas por metro (pl $\left.\mathrm{m}^{-1}\right)$.

\begin{tabular}{ccc}
\hline $\mathbf{P I ~}^{-1}$ & Rendimiento $\left(\mathbf{k g ~ h a}^{-1}\right)$ & $\mathbf{n}$ \\
\hline 3 & $2137,19 \mathrm{~A}$ & 12 \\
3.83 & $2285,73 \mathrm{~A}$ & 12 \\
2.17 & $2621,69 \mathrm{~A}$ & 12 \\
\hline
\end{tabular}

Test:LSD Fisher Alfa $=0,05$ DMS $=634,24435$

Medias con una letra común no son significativamente diferentes $(p>0,05)$.

Tabla $\mathbf{N}^{\circ}$ 4: Rendimiento medio $\left(\mathrm{kg} \mathrm{ha}^{-1}\right)$ en función de la dosis de urea aplicada $\left(\mathrm{kg} \mathrm{ha}^{-1}\right)$.

\begin{tabular}{ccc}
\hline Urea $\left(\mathbf{k g ~ h a}^{-1}\right)$ & Rendimiento $\left(\mathbf{k g ~ h a}^{-1}\right)$ & $\mathbf{n}$ \\
\hline 0 & $1737,54 \mathrm{~A}$ & 12 \\
150 & $2531,14 \mathrm{~B}$ & 12 \\
100 & $2775,92 \mathrm{~B}$ & 12 \\
\hline
\end{tabular}

Test:LSD Fisher Alfa $=0,05$ DMS $=534.17326$

Medias con una letra común no son significativamente diferentes $(p>0,05)$. 
Tabla $\mathbf{N}^{\mathbf{0}}$ 5: Rendimiento medio en función de la dosis de urea (kg urea $\mathrm{ha}^{-1}$ ) para cada cantidad de plantas por metro.

\begin{tabular}{ccccc}
\hline \multirow{2}{*}{$\begin{array}{c}\text { Plantas } \\
\text { por metro }\end{array}$} & $\mathbf{0}$ & $\mathbf{k g}$ urea ha & \\
\cline { 2 - 5 } & $\mathbf{0}$ & $\mathbf{1 0 0}$ & $\mathbf{1 5 0}$ & $\mathbf{n}$ \\
\hline 2.17 & $2282.98 \mathrm{C}$ & $3349.33 \mathrm{~A}$ & $2232.75 \mathrm{~A}$ & 4 \\
3 & $1233.75 \mathrm{~A}$ & $2435.53 \mathrm{~A}$ & $2742.27 \mathrm{~A}$ & 4 \\
3.83 & $1695.89 \mathrm{~B}$ & $2542.89 \mathrm{~A}$ & $2618.39 \mathrm{~A}$ & 4 \\
DMS & 389.47439 & 1149.65227 & 1024.75154 & \\
\hline
\end{tabular}

Alfa $=0,05$ DMS $=389,47439$

Medias con una letra común no son significativamente diferentes en cada columna .Test: LSD Fisher $(p>0,05)$.

Tabla $\mathbf{N}^{\circ}$ 6: Interacción dosis de urea aplicada y plantas logradas por metro.

\begin{tabular}{lcccc}
\hline F.V & SC & gl & F & p-valor \\
\hline Modelo & 3294226,73 & 4 & 1,47 & 0,2366 \\
Plantas por metro*Dosis urea & 3294226,73 & 4 & 1,47 & 0,2366 \\
Error & 17426748,95 & 31 & & \\
Total & 20720975,69 & 35 & & \\
\hline
\end{tabular}

bien, no refleja incrementos en los rendimientos por incremento de la densidad. Se observa que, a pesar del aumento en la densidad, sin fertilización los rendimientos no superan los $2800 \mathrm{~kg} \mathrm{ha}^{-1}$, en tanto si es posible alcanzar rendimientos superiores a $2800 \mathrm{~kg} \mathrm{ha}^{-1}$, únicamente con fertilización nitrogenada de 100 y $150 \mathrm{~kg} \mathrm{ha}^{-1}$ de urea con incrementos en la densidad de siembra.

A medida que aumenta la cantidad de plantas en el metro disminuye el peso de mil semillas (Figura 4). Igual comportamiento observó Hernandez (1983), citado por Aguirrezabal et al. (2001) para el cultivar Dekalb G-97.

Existe una relación directa entre la cantidad de plantas por metro en el surco y la altura de

Para dosis de $100 \mathrm{~kg}$ de urea por ha, no existen diferencias significativas en el rendimiento a pesar que los mayores rendimientos $3349.33 \mathrm{~kg} \mathrm{x} \mathrm{ha}^{-1} \mathrm{se}$ obtuvieron con la menor cantidad de plantas por metro (Tabla $\mathrm{N}^{\mathrm{o}}$ 5). Para dosis de $150 \mathrm{~kg}$ de urea ha ${ }^{-1}$ no hubo diferencia significativa entre tratamientos, a pesar de obtener los más altos rendimientos $(2742.27 \mathrm{~kg}$ $\mathrm{ha}^{-1}$ ) con 3 plantas por metro (Tabla $\mathrm{N}^{\mathrm{o}} 5$ ).

No existe interacción entre la dosis de urea aplicada y la cantidad de plantas por metro logradas como lo muestra la Tabla $\mathbf{N}^{0} \mathbf{6}$.

En la Figura $\mathbf{N}^{0} 3$ se observa que la relación entre los rendimientos y la cantidad de plantas por $\mathrm{m}$ desde $1.63 \mathrm{pl} \mathrm{m}^{-1}$ hasta $4.38 \mathrm{pl} \mathrm{m}^{-1}$ no es lineal, más

Rendimiento a $11 \%(\mathrm{~kg} / \mathrm{ha})-150$

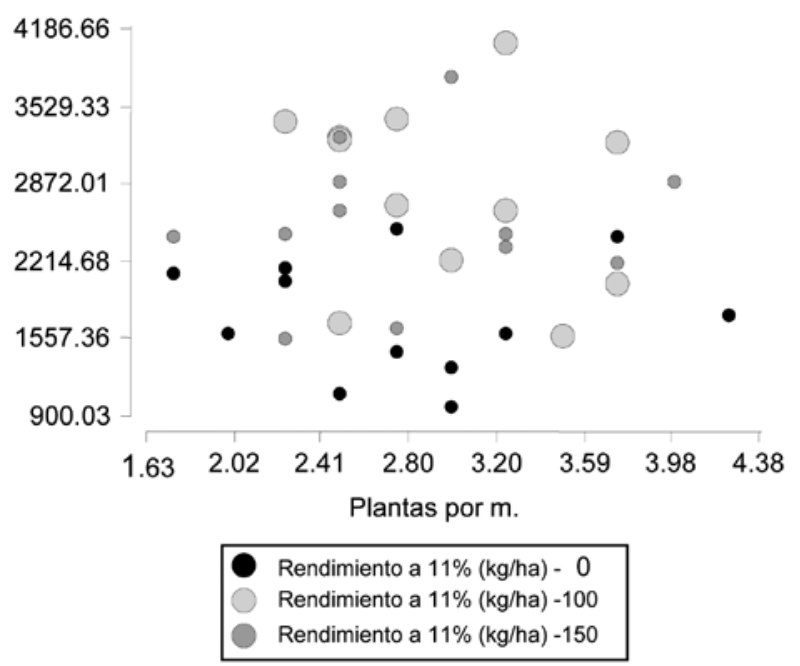

Figura $\mathbf{N}^{0} 3$. Relación plantas por metro y rendimiento. plantas de girasol, Figura $\mathbf{N}^{\circ} \mathbf{5}$ por efecto de la competencia por luz. En este sentido, Vranceanu, et al.

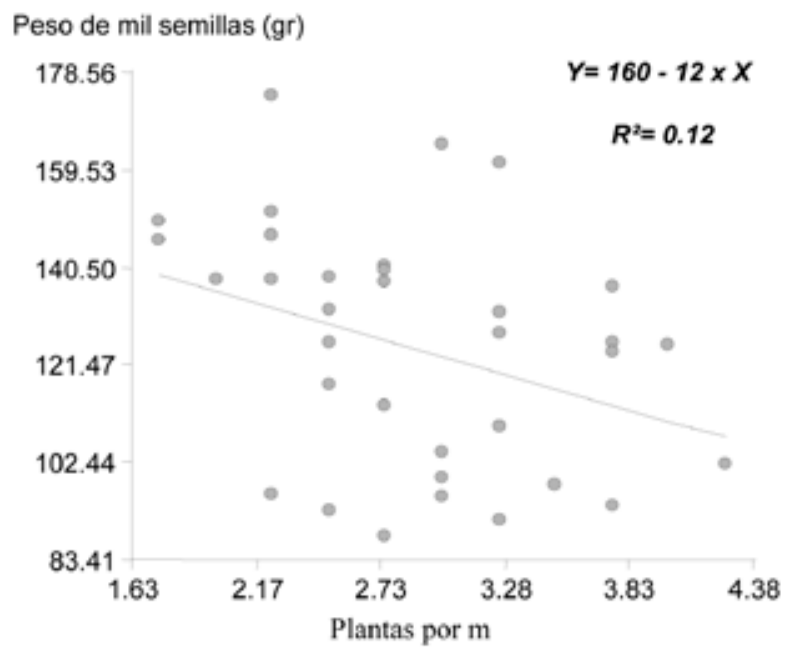

Figura $N^{\circ}$ 4. Relación entre plantas por metro en el surco y peso de mil semillas.

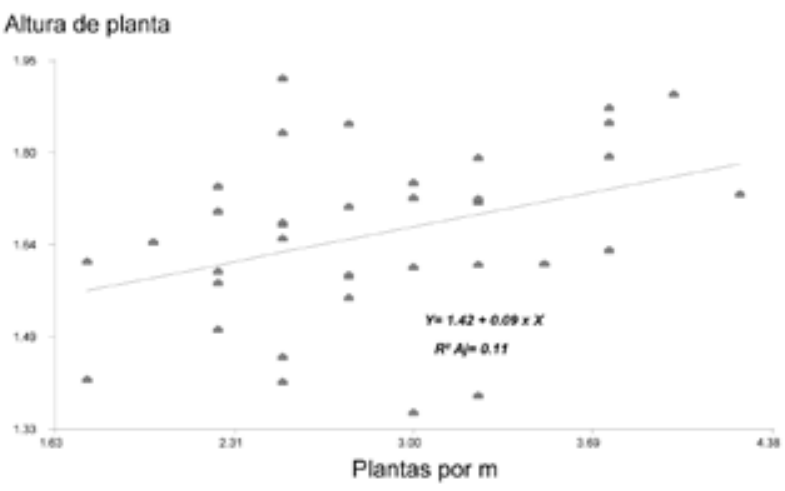

Figura $N^{0}$ 5. Regresión entre altura de plantas y plantas por $\mathrm{m}$. 
Tabla $\mathbf{N}^{0}$ 7. Eficiencia del uso del agua y de urea.

\begin{tabular}{|c|c|c|c|c|c|c|c|}
\hline $\begin{array}{c}\text { Rendimiento } \\
\left(\mathrm{kg} \mathrm{ha}^{-1}\right)\end{array}$ & $\begin{array}{c}\text { Dosis } \\
\text { urea } \\
\left(\mathrm{kg} \mathrm{ha}^{-1}\right)\end{array}$ & $\begin{array}{l}\text { Agua total } \\
\text { en el ciclo } \\
(\mathrm{mm})\end{array}$ & $\begin{array}{l}\text { kg grano } \\
\text { mm agua }^{-1}\end{array}$ & $\begin{array}{c}\text { Incremento } \\
\text { de rendimiento } \\
\left(\mathrm{kg} \mathrm{ha}^{-1}\right)\end{array}$ & $\begin{array}{l}\% \text { de incremento } \\
\text { de rendimiento } \\
\text { respecto a testigo }\end{array}$ & $\begin{array}{l}\text { kg grano } \\
\text { logrado kg urea } \\
\text { aplicado }^{-1}\end{array}$ & $\begin{array}{c}\text { Incremento de } \\
\text { rendimiento kg } \\
\text { de urea }^{-1}\end{array}$ \\
\hline $\begin{array}{c}1737 \\
\pm 499.61\end{array}$ & 0 & 360 & 4.83 & 0 & 0 & 0 & 0 \\
\hline $\begin{aligned} & 2775 \\
+ & 777.22\end{aligned}$ & 100 & 360 & 7.71 & 1038 & 59.7 & 27.75 & 10.38 \\
\hline $\begin{array}{c}2531 \\
\pm 622.21\end{array}$ & 150 & 360 & 7.03 & 794 & 45.7 & 16.87 & 5.29 \\
\hline
\end{tabular}

(1982), citado por Ortegón y Díaz (1999) mencionan que este aumento estaría asociado a competencia por luz y que podrían provocar mayor susceptibilidad a enfermedades.

En la Figura $\mathbf{N}^{\mathbf{0}} \mathbf{6}$ se observa que existe una relación inversa entre la cantidad de plantas por metro y el diámetro del capítulo. Esto fue observado por (Mathers y Stewart, 1982; Mohammad, Akhtar y Shakoor, 1986; Vannozzi, Salera y Baldini, 1990; Ujjanaiah et al., 1993), citado por Ortegón y Díaz (1999).

En la Figura $\mathbf{N}^{\mathbf{0}} \mathbf{7}$ se observa la relación positiva entre la dosis de urea aplicada y el diámetro del capítulo, esto también fue observado por Narwak y Malik (1985) quienes mencionan existe una relación positi-

Diametro de capítulo

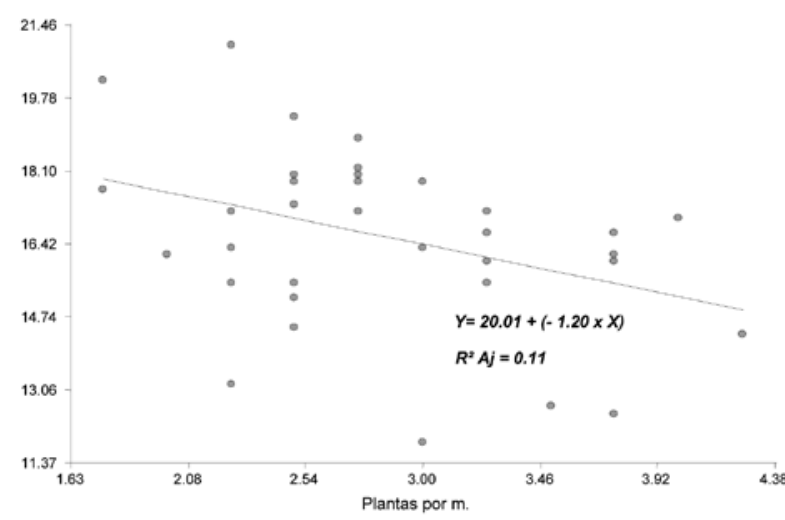

Figura $\mathbf{N}^{\mathbf{0}}$ 6. Regresión entre diámetro de capítulo y plantas por metro.

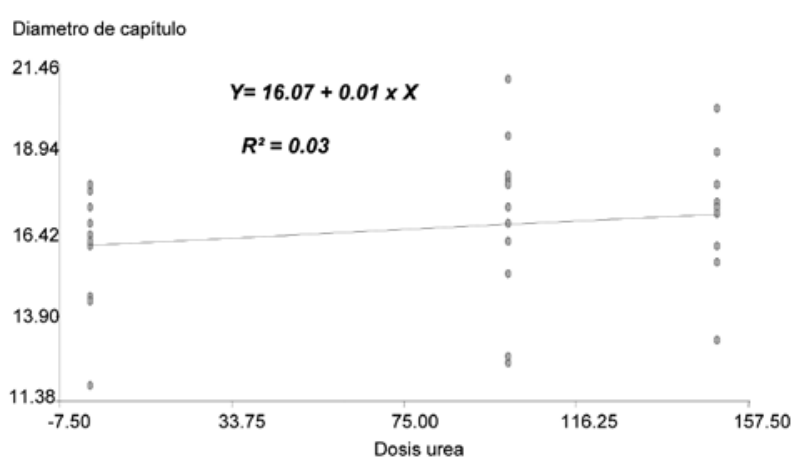

Figura $\mathbf{N}^{\mathbf{0}}$ 7. Regresión entre dosis de urea y diámetro de capítulo. va entre la dosis de urea y el diámetro de capítulo, el peso de mil semillas y el rendimiento por planta, con dosis superiores a $60 \mathrm{~kg}$ de urea por ha.

En la Figura $\mathbf{N}^{\mathbf{0}} \mathbf{8}$ se puede observar que el incremento en el rendimiento de girasol, responde a una ecuación de regresión polinómica con un máximo a los $100 \mathrm{~kg}$ de urea aplicada, continuando el descenso con $150 \mathrm{~kg}$ de urea aplicado al cultivo en inicio de floración.

Como lo muestra la Tabla $\mathbf{N}^{\mathbf{0}}$ 7, existe un incremento en la eficiencia del uso del agua, pasando de $4.83 \mathrm{~kg}$ de grano $\mathrm{mm}$ de agua ${ }^{-1}$ en el Testigo sin fertilización a $7.71 \mathrm{~kg}$ de grano $\mathrm{mm}$ de agua ${ }^{-1}$ con $100 \mathrm{~kg}$ de urea, disminuyendo luego a $7.03 \mathrm{~kg}$ de grano $\mathrm{mm}$ de agua $^{-1}$ con $150 \mathrm{~kg}$ de urea. En tanto, el incremento de rinde respecto a los $\mathrm{kg}$ de urea agregados pasan de $10.38 \mathrm{~kg}$ de grano $\mathrm{x} \mathrm{kg}$ de urea-1 con $100 \mathrm{~kg} \mathrm{ha}^{-1} \mathrm{a}$ $5.29 \mathrm{~kg}$ de grano $\mathrm{x} \mathrm{kg}$ de urea ${ }^{-1}$ a dosis de $150 \mathrm{~kg} \mathrm{ha}^{-1}$.

Los $360 \mathrm{~mm}$ de agua utilizada por el cultivo en el ciclo, indican que los rendimientos alcanzados estarían entre los rendimientos potenciales alcanzados por el cultivo, de acuerdo a lo mencionado por Halvorson et al. (1999) como respuesta a la labranza y fertilización nitrogenada.

Quiroga et al. (2002) indican de 200 a $2800 \mathrm{~kg} \mathrm{ha}^{-1}$ de incrementos de rendimiento en girasol fertilizado con $40 \mathrm{~kg} \mathrm{~N} \mathrm{ha}^{-1}$ y mencionan que el agua y el $\mathrm{N}$ (ni-

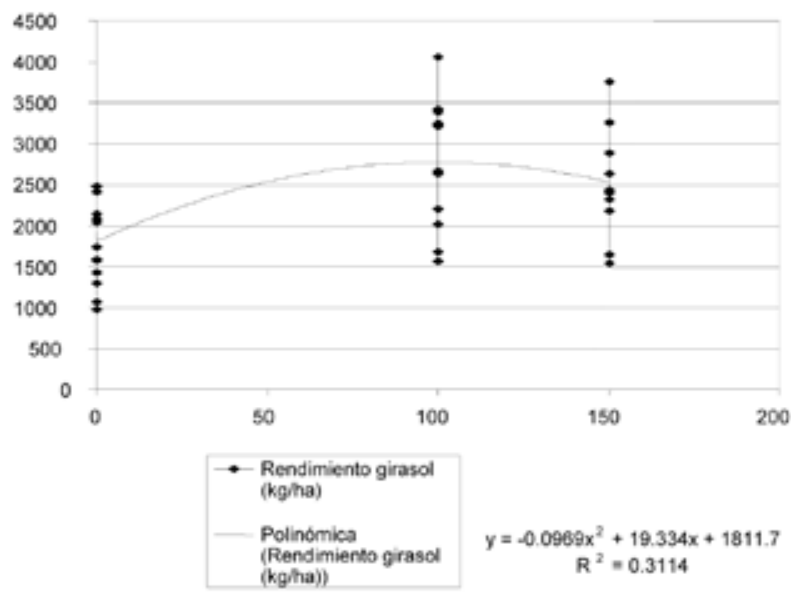

Figura $\mathbf{N}^{\mathbf{0}} \mathbf{8}$. Ecuación de regresión polinómica, dosis de urea, rendimiento de girasol. 
tratos a la siembra y aportados por el suelo durante el cultivo) son los principales factores a tener en cuenta por su incidencia en la productividad. El contenido de agua a la siembra, si bien resultó dependiente de características de los suelos (textura, espesor, compactaciones) fue influenciado significativamente por prácticas de manejo del agricultor (cultivo antecesor, barbecho, labranza).

\section{CONCLUSIONES}

No existen diferencias significativas en los rendimientos en función de la cantidad de plantas por metro, a pesar que los más altos rendimientos 2621.69 $\mathrm{kg} \mathrm{ha}^{-1}$ se dan con la menor cantidad de plantas en el metro (2.17 $\left.\mathrm{pl} \mathrm{metro}^{-1}\right)$.

El agregado de $100 \mathrm{~kg}$ de urea al inicio de floración incorporada al surco permitió incrementar los rendimientos de girasol en $59.7 \%$ con $100 \mathrm{~kg}$ de urea por ha-1.

Existe una correlación positiva entre el diámetro de capítulo, peso de mil semillas, altura de plantas dosis de urea y el rendimiento del cultivo y negativa para el rendimiento y la cantidad de plantas por metro.

La fertilización mejoró la eficiencia del uso del agua hasta los $100 \mathrm{~kg}$ de urea ha ${ }^{-1}$, pasando de 4.83 $\mathrm{kg}$ de grano en el Testigo sin urea a $7.71 \mathrm{~kg}$ de grano por $\mathrm{mm}$ de agua consumida en el ciclo del cultivo, bajando luego a $7.03 \mathrm{~kg}$ de grano por $\mathrm{mm}$ de agua consumida para $150 \mathrm{~kg}$ de urea ha-1.

\section{AGRADECIMIENTOS}

Al Proyecto Regional de INTA CHFOR 410091 Manejo de cereales y oleaginosas en labranza conservacionista en Chaco y Formosa.

Al Ing. Gerardo Quintana, EEA INTA Las Breñas, por sus aportes en la revisión de este artículo.

\section{BIBLIOGRAFÍA}

Balzarini M.G., Di Rienzo J.A. Info-Gen versión 2012. FCA, Universidad Nacional de Córdoba, Argentina. URL http://www.info-gen.com.ar
Ghanavati, N. A.; Nahavandi, E.; Ghaderi, A. 1981. Breeding sunflower for semiarid regions. The Journal of Agricultural Science. 96. PP 447 a 450. doi:10.1017/ S0021859600066211.

Halvorson, A..D.; Black A.L.; Krupinsky, J.M.; Merill, s. D.; Tanaka, D.L.. 1999. Sunflower response to tillage and nitrogen fertilization Ander intensive cropping in a wheat rotation. Agronomy Journal. Vol. $91 \mathrm{~N}^{\circ} 4$. Pag. 637 - 642. DOI: 10.2134/agronj1999.914637x.

Hernandez (1983), In Aguirrezabal, L. A. N. et al (2001). Girasol. Aspectos fisiológicos que determinan el rendimiento. Unidad Integrada Balcarce INTA - UNMDP. Mar del Plata, Bs. As., Argentina. 177 páginas.

Herrera, G. 2011. Boletín Agrometeorológico Anual 2011 (temperaturas y precipitaciones). Estación Agrometeorológica "Delfino Juan Pallaoro". EEA INTA Las Breñas. Las Breñas, Chaco. http://anterior.inta.gov.ar/lasbrenas/info/boletines/meteorologia/Anual2011.pdf.

Melean, J. A. 2009. Manual para el cultivo del girasol. Serie $\mathrm{b}^{\circ}$ 20. INIA Venezuela. Maracay, Venezuela. 52 páginas. www.sian.inia.gob.ve/repositorio/noperiodicas/pdf/ Manual de girasol_dgtl.pdf.

Narwal S.S.; Malik, D.S.. 1985. Response of sunflower cultivars to plant density and nitrogen. The Journal of Agricultural Science. Volume 104. Issue 01. February. Pag 95 a 97. doi:http://dx.doi.org/10.1017/ S0021859600043021. On line: 27 March 2009.

Ortegon, A.S.; Díaz,A. 1999. Respuesta de cultivares de girasol a la densidad de población en dos ambientes. Agronomía Mesoamericana 10 (2) : 17-21.

Parra, R.; Sosa, M.A. 2002. La soja y el girasol en el Noreste Argentino. IDIA XXI. Año II No 3. Ediciones INTA. Bs. As. Páginas 41 a 47.

Parra, R.; Longhi, T. 2010. Red nacional de cultivares comerciales de girasol de INTA. Ciclos 2008/2009 y 2009/210. 76 Páginas. ASAGIR Cuadernillo $\mathrm{N}^{\circ} 18$. www.asagir. org.ar/asagir2008/archivos_arcp/201096113942.pdf.

Quiroga, A.; Bono A.; Corro Molas, A. 2002. Aspectos nutricionales del girasol en la región semiárida y subhúmeda pampeana. IDIA XXI. Año II No 3 . Ediciones INTA. Bs. As. Página 128 a 134. 